\title{
Saturable Absorption and Modulation Characteristics of Laser with Graphene Oxide Spin Coated on ITO Substrate
}

\author{
Xin Li, ${ }^{1}$ Haikun Zhang, ${ }^{1}$ Peiji Wang, ${ }^{1}$ Guiqiu Li, ${ }^{2}$ Shengzhi Zhao, ${ }^{2}$ \\ Jing Wang, ${ }^{1}$ and Lijuan Chen ${ }^{1}$ \\ ${ }^{1}$ School of Physics and Technology, University of Jinan, Jinan, Shandong 250022, China \\ ${ }^{2}$ School of Information Science and Engineering, Shandong University, Jinan, Shandong 250100, China \\ Correspondence should be addressed to Xin Li; sps_lix@ujn.edu.cn
}

Received 24 January 2014; Revised 27 February 2014; Accepted 28 February 2014; Published 7 April 2014

Academic Editor: Xiang Wu

Copyright (C) 2014 Xin Li et al. This is an open access article distributed under the Creative Commons Attribution License, which permits unrestricted use, distribution, and reproduction in any medium, provided the original work is properly cited.

The graphene oxide (GO) thin film has been obtained by mixture of GO spin coated on substrate of indium tin oxide (ITO). The experiment has shown that continuous-wave laser is modulated when the graphene oxide saturable absorber (GO-SA) is employed in the $1064 \mathrm{~nm}$ laser cavity. The shortest pulse width is $108 \mathrm{~ns}$ at the pump power of $5.04 \mathrm{~W}$. Other output laser characteristics, such as the threshold pump power, the repetition rate, and the peak power, have also been measured. The results have demonstrated that graphene oxide is an available saturable absorber for $1064 \mathrm{~nm}$ passive Q-switching laser.

\section{Introduction}

For recent years, graphene has attracted significant attention for its remarkable mechanical and physical properties $[1,2]$. It is the building block nanomaterial of carbon nanotubes and graphite, a two-dimensional layer with carbon atoms arranged in a honey-comb lattice [3]. This feature has resulted in diverse applications in real world, such as plasmon wave guiding [4] and photodetectors [5]. Moreover, it is a potential candidate to enhance or enable the biomedical, chemical, and industrial processes $[6,7]$. The very important application is that it can provide the nonlinear saturable absorption covering a wide wavelength range $[8,9]$, due to its point band gap structures [10] combined with Pauli blocking [11]. There already have been some researches on nanosecond and picosecond lasers employing graphene as the saturable absorber [12-15]. The distribution of graphene sheet and its derivatives into polymers has been widely considered for the fabrication of nanocomposites with enhanced properties $[16,17]$. It can enhance the strength of the parent material. Compared with the pure polymer, the graphene polymer has better mechanical, thermal, electrical, gas barrier, and flame retardant properties.
Although the graphene has so many advantages and applications, the distribution of graphene sheets in the polymer matrix, as well as the relatively weak interfacial bonding with the polymer chain, limits the improvement of the physicochemical properties for the nanocomposite materials [18]. Moreover, the point band gap structure in graphene restricts it to application in nanoelectronics. As a potential alternative to graphene, graphene oxide (GO), an atomic sheet of carbon by analogy to graphene, has been discovered recently [19]. It is significantly more compatible with polymers. It has carboxylic groups at its edges and phenol and epoxide groups on its basal plane. The adsorption of oxygen opens up the band gap in the graphene. It is possible that mass of graphene and some of the graphene saturable absorbers can be obtained by reducing the stable GO $[20,21]$. Indium tin oxide (ITO) is a practical coated substrate for graphene in addition to the usual used quartz or $\mathrm{SiC}$ [22]. This substrate is a solid solution of indium oxide $\left(\mathrm{In}_{2} \mathrm{O}_{3}\right)$ and tin oxide $\left(\mathrm{SnO}_{2}\right)$, and it is widely employed in transparent conducting oxides due to the electrical conductivity and optical transparency.

For the above-mentioned reasons, we have prepared the graphene oxide thin films by mixture of GO spin coated on substrate of indium tin oxide (ITO). The transmissivity has 


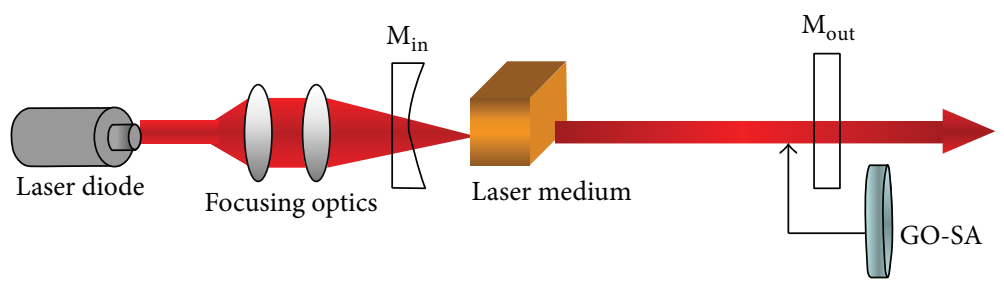

FIGURE 1: The experimental setup.

been measured, and it is around $76 \%$ at $1064 \mathrm{~nm}$ wavelength. We locate the GO-SA in the laser cavity and realize the Q-switched modulation. The output laser characteristics, such as the threshold pump power, the pulse width, the repetition rate, and the peak power, have been measured. The shortest pulse width of $108 \mathrm{~ns}$ has been obtained at the pump power of $5.04 \mathrm{~W}$. In this paper, the results have demonstrated that graphene oxide is an available saturable absorber for $1064 \mathrm{~nm}$ wavelength and it can effectively obtain the passive Q-switching pulse laser. The passively Q-switched laser performance of GO-SA is comparable with that obtained by graphene saturable absorber.

\section{Materials and Methods}

2.1. Preparation of the Graphene Oxide Saturable Absorber. The graphene oxide saturable absorber is obtained by spin coated mixture of GO on ITO substrate. The first step is to prepare the mixture of GO. The graphite and $\mathrm{NaNO}_{3}$ were mixed with the ratio of $2 / 1$, and then $\mathrm{H}_{2} \mathrm{SO}_{4}$ with the concentration of $95 \%$ was mixed together. $300 \mathrm{~g} \mathrm{KMnO}_{4}$ was subsequently injected into the suspension by inches. The injection should be together with nonstop agitation. The processes above were carried out in a flask and cooled in ice bath to avoid overheating. Then, the suspension was stirred at room temperature by machine lasting for two hours. With the distilled water being slowly added in the suspension, the bright brown suspension was observed to become yellow in color, caused by the temperature increasing to $90^{\circ} \mathrm{C}$. Then, the suspension should be sequentially stirred for twelve hours and treated with $30 \% \mathrm{H}_{2} \mathrm{O}_{2}$ to reduce the $\mathrm{KMnO}_{4}$ and $\mathrm{MnO}_{2}$. After that, the suspension was washed with hydrochloric of low concentration, then with distilled water. The last step was to centrifuge the suspension and then dry it under vacuum condition. The vital procedure of graphene oxide preparation is purification. There are still some salts and acids residues in GO powders, so they have a brown dispersion when the black synthesized graphene oxides (GOs) powders were suspended in water. Ultrapure Milli-Q water was used in order to effectively dialyze in all experiments. The GO in the brown dispersion was exfoliated and centrifuged. After powerful shaking, the dispersion in the vial was put in a high-temperature water bath. Finally, the brown-colored dispersion turned black.

We used the spin coating method to obtain a uniform thin liquid film composed of graphene oxide. The thickness of membrane depended on speed, rheology of solidifying liquid, and gas conditions. We have prepared the GO-SA by employing the spin coating method. The best films were obtained using spin coating from the graphene oxide colloid $\left(1.0 \mathrm{mg} \mathrm{mL}^{-1}\right)$ at ambient temperature condition and the spin-coating speed was $800 \mathrm{rpm}$. Then, the graphene oxide saturable absorber spin coated on ITO was obtained. Its transmissivity of different wavelengths is measured using UV-Vis adsorption spectroscopy. UV-Vis adsorption spectroscopy measurement was obtained using a Hitachi U-4100 spectrophotometer. The GO-SA can be used in the wide wavelength range from 1 to $2 \mu \mathrm{m}$. The transmissivity is $76 \%$, $74 \%, 73 \%$, and $62 \%$, corresponding to the wavelengths of $1.06 \mu \mathrm{m}, 1.34 \mu \mathrm{m}, 1.5 \mu \mathrm{m}$, and $2 \mu \mathrm{m}$, respectively.

2.2. Testing Experimental Setup. The saturable absorption characteristics of the graphene oxide have been operated in a laser-diode-pumped $\mathrm{Nd}: \mathrm{YVO}_{4}$ laser cavity at $1064 \mathrm{~nm}$. The arrangement of the experimental equipment is demonstrated in Figure 1. As is shown, a simple flat-concave cavity is employed. The whole length is about $6.5 \mathrm{~cm}$. The laser medium is pumped by a commercially used fiber-coupled laser-diode (FAP-I system, Coherent Inc., USA). Its output beam is $808 \mathrm{~nm}$ lasers with the maximum output power of $30 \mathrm{~W}$. The pump beam is focused into the laser crystal by a focusing optical system with a spot size of $400 \mu \mathrm{m}$. The laser oscillations operate between two coated mirrors. The input mirror $M_{\text {in }}$ is antireflection-coated at $808 \mathrm{~nm}$ on the entrance face, high-transmission-coated at $808 \mathrm{~nm}$, and highreflection-coated at $1.06 \mu \mathrm{m}$ on the other side, with the curvature radius of $200 \mathrm{~mm}$. The output coupler $M_{\text {out }}$ is coated with transmissions $(T)$ of $10 \%$ and $15 \%$ at $1.06 \mu \mathrm{m}$, with two plane end surfaces. The graphene oxide saturable absorber is placed close to the output coupler. An a-cut $\mathrm{Nd}: \mathrm{YVO}_{4}$ crystal with 1.0 at. $\% \mathrm{Nd}^{3+}$-doped is employed as the laser gain medium and located near the input mirror. Its dimensions are $3 \times 3 \times 10 \mathrm{~mm}^{3}$ and both end sides are high-transmissioncoated at $808 \mathrm{~nm}$ and antireflection-coated at $1.06 \mu \mathrm{m}$. The laser crystal is mounted in a copper heat-sink cooled by water, in order to keep its temperature at $20^{\circ} \mathrm{C}$ and dissipate the heat deposition. The output characteristics are measured by some detection equipment. The generated average output power is measured by the EPM 2000 energy/power meter (Molectron Detector Inc., USA). The pulse width and repetition rate of the laser pulses are measured by a photoelectric detector and a TED 6208 digital oscilloscope $(500 \mathrm{MHz}$ bandwidth, Tektronix Inc., USA).

\section{Results and Discussion}

The experimental setup is a flat-concave laser resonator. We choose $T=10 \%$ and $15 \%$ because low transmission of 


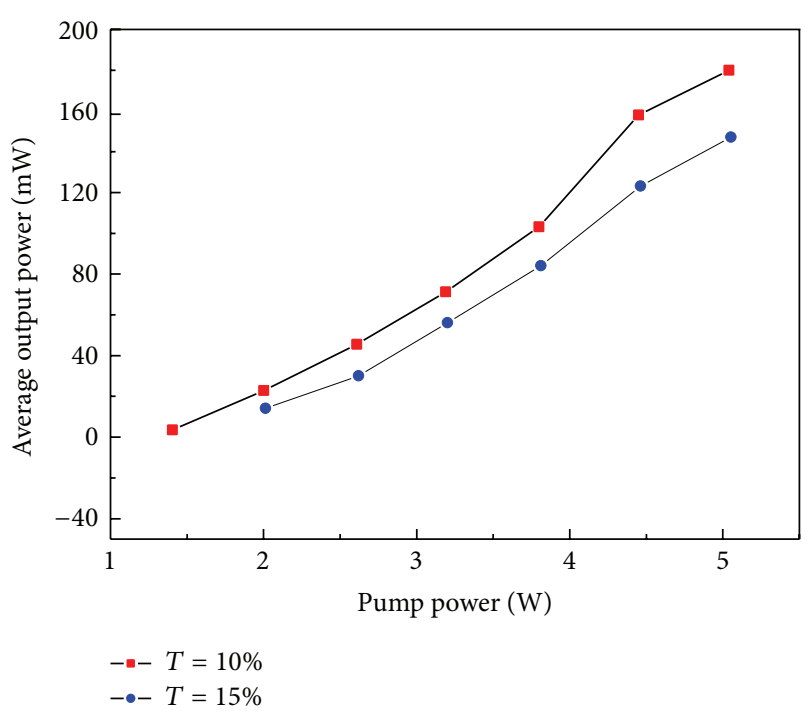

FIGURE 2: Average output power versus pump power.

TABLE 1: The continuous-wave output power.

\begin{tabular}{lccccccc}
\hline $\begin{array}{l}\text { Pump power } \\
\text { (W) }\end{array}$ & 0.235 & 1.405 & 2.61 & 3.8 & 4.45 & 5.04 & 6.2 \\
$\begin{array}{l}\text { Output power } \\
\text { (W) }\end{array}$ & 0.021 & 0.442 & 0.934 & 1.45 & 1.69 & 2.24 & 2.71 \\
\hline
\end{tabular}

output mirror can hold high photon number density in the resonant, so the laser performance is better expected. Two transmissions are employed for the purpose of comparison. The continuous-wave laser operation at $1064 \mathrm{~nm}$ should be operated at first. The incident pump power of $808 \mathrm{~nm}$ laser continuously increases. The laser begins to radiate at the pump power of $235 \mathrm{~mW}$, with the output power being $0.021 \mathrm{~W}$, as shown in Table 1 . The maximum power is $2.71 \mathrm{~W}$ at the pump power of $6.2 \mathrm{~W}$, with the optical-optical conversion efficiency of $43.7 \%$.

In order to detect the saturable absorption characteristics of the GO-SA, it is located near the output mirror in the cavity. Then, the threshold pump powers increase to $1.37 \mathrm{~W}$ and $1.62 \mathrm{~W}$ corresponding to the output transmissivity of $10 \%$ and $15 \%$. The larger threshold pump powers demonstrate the absorption of low power laser. The threshold pump powers are much lower than those of laser passively Q-switched with grapheme saturable absorber (3.14 W with $\mathrm{Nd}: \mathrm{LuVO}_{4}[23]$ ). Figure 2 has shown the average output power versus pump power. It is obvious that the largest output power is obtained by $T=10 \%$. It can be seen that the maximum output power is measured to be $180 \mathrm{~mW}$, obtained at the pump power of $5.04 \mathrm{~W}$. The laser average output power of $T=15 \%$ is always lower than that obtained by $T=10 \%$.

The passive Q-switched operation has been realized once the pump power reaches the threshold. A typical temporal pulse shape with the pulse width of $108 \mathrm{~ns}$ has been depicted in Figure 3.

As is shown in Figure 4, in which the pulse width versus pump power has been demonstrated, the pulse widths

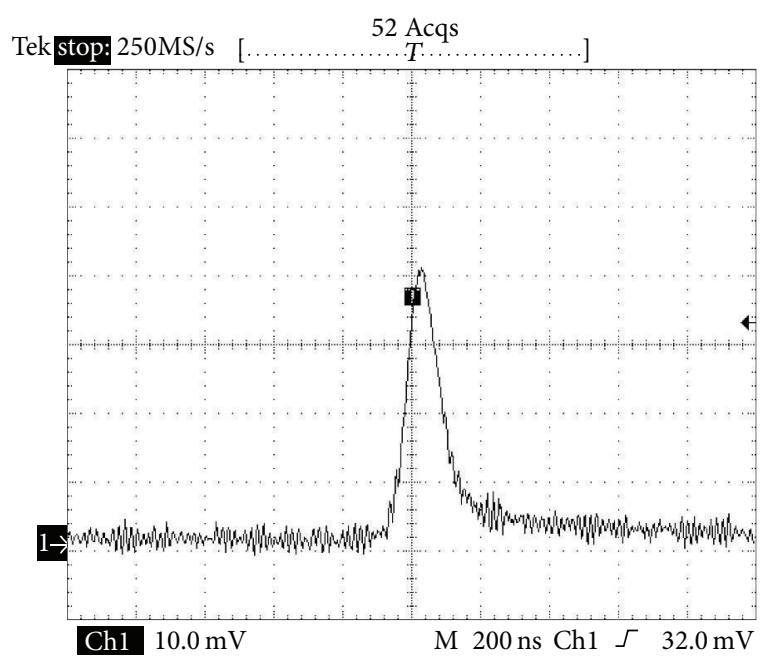

FIGURE 3: Temporal profile of the single pulse with pulse width of $108 \mathrm{~ns}$.

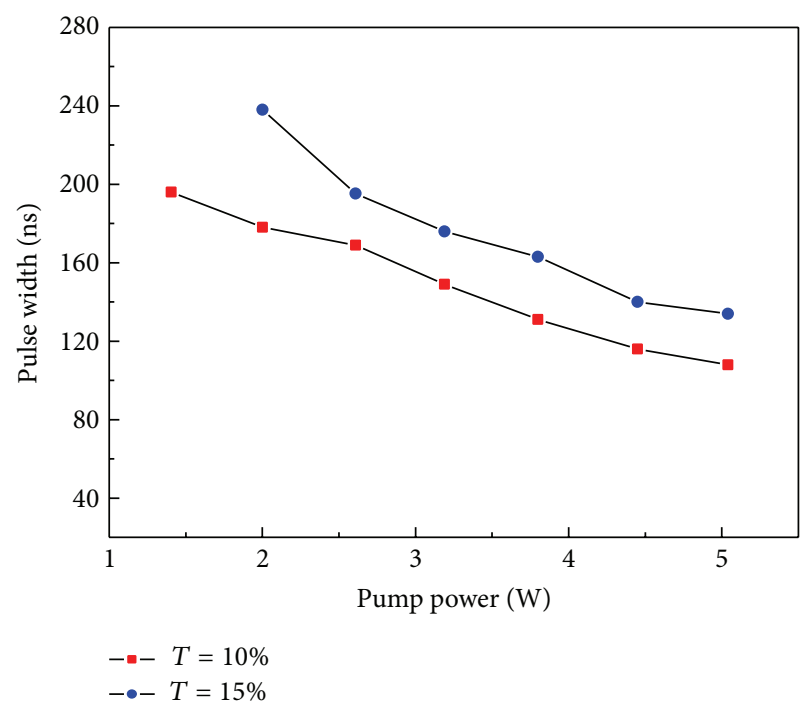

FIGURE 4: Pulse width versus pump power.

monotonously decrease along with the increase of pump power. The shortest pulse width is $108 \mathrm{~ns}$ obtained by $T=10 \%$ at the pump power of $5.04 \mathrm{~W}$. The data of $T=15 \%$ are larger than those of $T=10 \%$ at each pump power. The pulse width for $T=15 \%$ continuously reduces from $240 \mathrm{~ns}$ to $134 \mathrm{~ns}$ with the pump power increasing from $2 \mathrm{~W}$ to $5.04 \mathrm{~W}$. The passively Q-switched laser performance of GO-SA is comparable with that obtained by graphene saturable absorber. We obtain the shortest pulse width at the pump power of $5.04 \mathrm{~W}$, and the shortest pulse width obtained by graphene SA is $161 \mathrm{~ns}$ at the pump power of $12.9 \mathrm{~W}$ [14]. Also, the pulse width obtained by GO-SA is shorter than half of that obtained by graphene SA with Nd:YAG (260 ns) [15].

Figure 5, in which the variation of pulse repetition rate versus pump power is displayed, has demonstrated that the repetition rates for $T=10 \%$ and $T=15 \%$ increase with 


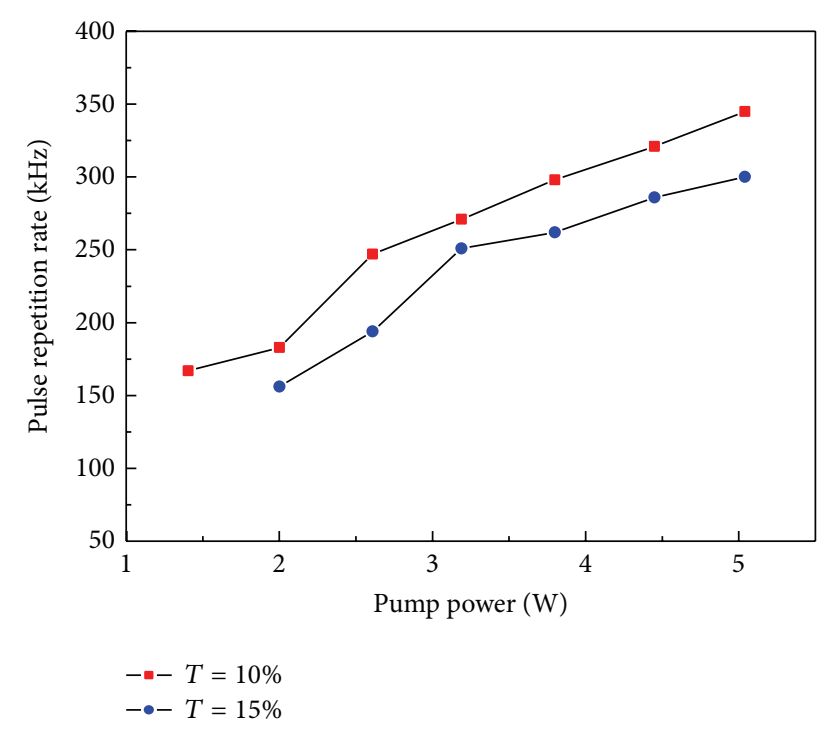

FIgURE 5: Pulse repetition rate versus pump power.

the accretion of incident pump power. At the pump power of $2 \mathrm{~W}$, the value for $T=10 \%$ and $T=15 \%$ is $183 \mathrm{kHz}$ and $156 \mathrm{kHz}$, respectively. The largest repetition rate is $345 \mathrm{kHz}$, obtained by $T=10 \%$ at the pump power of $5.04 \mathrm{~W}$, while the maximum for $T=15 \%$ is $300 \mathrm{kHz}$. With graphene $\mathrm{SA}$, the repetition rate is $167 \mathrm{kHz}$ when the pulse width reaches the minimum [15].

By mathematical computation, single pulse energy $E$ and the peak power $P_{\text {peak }}$ can be calculated out $\left(E=P_{\text {out }} / F\right.$ and $P_{\text {peak }}=E / W$, where $P_{\text {out }}$ is the average output power, $F$ is the pulse repetition rate, and $W$ is the pulse width). Both the pulse energy and the peak power of $T=10 \%$ or $T=$ $15 \%$ monotonously increase along with the pump power. The values for $T=10 \%$ are always larger than those of $T=15 \%$. The largest pulse energy of $0.52 \mu \mathrm{J}$ and the highest peak power of $4.8 \mathrm{~W}$ are obtained by $T=10 \%$, while the maximums for $T=$ $15 \%$ are $0.49 \mu \mathrm{J}$ and $3.7 \mathrm{~W}$ under the same conditions.

Besides GO-ITO saturable absorber, other GO composite material absorbers also show optical absorption ability. There is report on mode-locked laser operation with grapheme oxide/polyvinyl alcohol composite material, in which the absorber is employed as the output coupler. The average output power of $680 \mathrm{~mW}$ and the pulse duration of $12 \mathrm{ps}$ are obtained [24]. Reduced graphene oxide-Pani hybrid has optical absorption in UV-Visible spectrum. The GO exhibits a broad absorption which is continuously decreasing to $800 \mathrm{~nm}$, whereas the hybrid in dimethyl formamide shows an absorption maximum at $325 \mathrm{~nm}$, attributed to the $\pi-\pi^{*}$ transition, and a peak at $618 \mathrm{~nm}$ due to the excitonic transition of polyaniline [25].

\section{Conclusions}

In conclusion, we have prepared the graphene oxide thin films by mixture of GO spin coated on substrate of indium tin oxide (ITO). The transmissivity has been measured and it is around $76 \%$ at $1064 \mathrm{~nm}$ wavelength. The experiment has shown that once the graphene oxide saturable absorber (GOSA) is employed in the $1064 \mathrm{~nm}$ laser cavity, the continuouswave laser is modulated. The output laser characteristics, such as the threshold pump power, the pulse width, the repetition rate, and the peak power, have been measured. The shortest pulse width of $108 \mathrm{~ns}$ is obtained at the pump power at $5.04 \mathrm{~W}$. The results have demonstrated that graphene oxide is an available saturable absorber for $1064 \mathrm{~nm}$ wavelength and it can effectively achieve passive Q-switching laser operation.

\section{Conflict of Interests}

The authors declare that there is no conflict of interests regarding the publication of this paper.

\section{Acknowledgments}

This work was supported by the Encouragement Foundation for Excellent Middle-aged and Young Scientist of Shandong Province (no. BS2013DX024), Doctoral Foundation of University of Jinan (UJN) (no. XBS1327), and a Project of Shandong Province Science and Technology Development Program (no. 2010G0020423).

\section{References}

[1] C. Ramirez, P. Miranzo, M. Belmonte et al., "Extraordinary toughening enhancement and flexural strength in $\mathrm{Si}_{3} \mathrm{~N}_{4}$ composites using graphene sheets," Journal of the European Ceramic Society, vol. 34, pp. 161-169, 2014.

[2] V. Vijayaraghavan, A. Garg, C. H. Wong, K. Tai, and S. S. Mahapatra, "Measurement of properties of graphene sheets subjected to drilling operation using computer simulation," Measurement, vol. 50, pp. 50-62, 2014.

[3] K. S. Novoselov, A. K. Geim, S. V. Morozov et al., "Electric field in atomically thin carbon films," Science, vol. 306, no. 5696, pp. 666-669, 2004.

[4] J. Christensen, A. Manjavacas, S. Thongrattanasiri, F. H. L. Koppens, and F. J. García de Abajo, "Graphene plasmon waveguiding and hybridization in individual and paired nanoribbons," ACS Nano, vol. 6, no. 1, pp. 431-440, 2012.

[5] F. Xia, T. Mueller, Y.-M. Lin, A. Valdes-Garcia, and P. Avouris, "Ultrafast graphene photodetector," Nature Nanotechnology, vol. 4, no. 12, pp. 839-843, 2009.

[6] X. H. Hu, X. H. Ma, H. P. Tan, and D. Li, "Preparation of watersoluble and biocompatible graphene," Nano-Micro Letters, vol. 8, no. 6, p. $277,2013$.

[7] J. An, Y. Q. Gou, C. X. Yang, F. D. Hu, and C. M. Wang, "Synthesis of a biocompatible gelatin functionalized graphene nanosheets and its application for drug delivery," Materials Science and Engineering: C, vol. 33, pp. 2827-2837, 2013.

[8] R. Alaee, M. Farhat, C. Rockstuhl, and F. Lederer, "A perfect absorber made of a graphene micro-ribbon metamaterial," Optics Express, vol. 20, Article ID 28017, 2012.

[9] S. Thongrattanasiri, F. H. L. Koppens, and F. J. García de Abajo, "Complete optical absorption in periodically patterned graphene," Physical Review Letters, vol. 108, no. 4, Article ID 047401, 2012. 
[10] Q. Bao, H. Zhang, Y. Wang et al., "Atomic-layer craphene as a saturable absorber for ultrafast pulsed lasers," Advanced Functional Materials, vol. 19, no. 19, pp. 3077-3083, 2009.

[11] Z. Sun, T. Hasan, F. Torrisi et al., "Graphene mode-locked ultrafast laser," ACS Nano, vol. 4, no. 2, pp. 803-810, 2010.

[12] Y. W. Song, S. Y. Jang, W. S. Han, and M. K. Bae, "Graphene mode-lockers for fiber lasers functioned with evanescent field interaction," Applied Physics Letters, vol. 96, Article ID 051122, 2010.

[13] J. L. Xu, X. L. Li, Y. Z. Wu, X. P. Hao, J. L. He, and K. J. Yang, "Graphene saturable absorber mirror for ultra-fast-pulse solidstate laser," Optics Letters, vol. 36, pp. 1948-1950, 2011.

[14] H. Yu, X. Chen, H. Zhang et al., "Large energy pulse generation modulated by graphene epitaxially grown on silicon carbide," ACS Nano, vol. 4, no. 12, pp. 7582-7586, 2010.

[15] M. Jiang, Z. Ren, Y. Zhang, B. Lu, L. Wan, and J. Bai, "Graphenebased passively Q-switched diode-side-pumped Nd:YAG solid laser," Optics Communications, vol. 284, no. 22, pp. 5353-5356, 2011.

[16] T. Ramanathan, A. A. Abdala, S. Stankovich et al., "Functionalized graphene sheets for polymer nanocomposites," Nature Nanotechnology, vol. 3, no. 6, pp. 327-331, 2008.

[17] D. Cai and M. Song, "Recent advance in functionalized graphene/polymer nanocomposites," Journal of Materials Chemistry, vol. 20, no. 37, pp. 7906-7915, 2010.

[18] O. K. Park, S. G. Kim, N. H. You, B. C. Ku, D. Hui, and J. H. Lee, "Synthesis and properties of iodo functionalized graphene oxide/polyimide nanocomposites," Composites B: Engineering, vol. 56, pp. 365-371, 2014.

[19] D. R. Dreyer, S. Park, C. W. Bielawski, and R. S. Ruoff, "The chemistry of graphene oxide," Chemical Society reviews, vol. 39, no. 1, pp. 228-240, 2010.

[20] Y. G. Wang, H. R. Chen, X. M. Wen, W. F. Hsieh, and J. Tang, "A highly efficient graphene oxide absorber for Q-switched $\mathrm{Nd}: \mathrm{GdVO}_{4}$ lasers," Nanotechnology, vol. 22, no. 45, Article ID 455203, 2011.

[21] J. Q. Zhao, Y. G. Wang, P. G. Yan et al., "Graphene-oxide-based Q-switched fiber laser with stable five-wavelength operation," Chinese Physics Letters, vol. 29, Article ID 114206, 2012.

[22] W. D. Tan, C. Y. Su, R. J. Knize, G. Q. Xie, L. J. Li, and D. Y. Tang, "Mode locking of ceramic Nd:yttrium aluminum garnet with graphene as a saturable absorber," Applied Physics Letters, vol. 96, no. 3, Article ID 031106, 2010.

[23] H. Yu, X. Chen, X. Hu et al., "Graphene as a Q-Switcher for neodymium-doped lutetium vanadate laser," Applied Physics Express, vol. 4, Article ID 022704, 2011.

[24] Y. G. Wang, H. R. Chen, W. F. Hsieh, and Y. H. Tsang, "Modelocked Nd: $\mathrm{GdVO}_{4}$ laser with graphene oxide/polyvinyl alcohol composite material absorber as well as an output coupler," Optics Communications, vol. 289, pp. 119-122, 2013.

[25] T. Remyamol, H. John, and P. Gopinath, "Synthesis and nonlinear optical properties of reduced graphene oxide covalently functionalized with polyaniline," Carbon, vol. 59, pp. 308-314, 2013. 

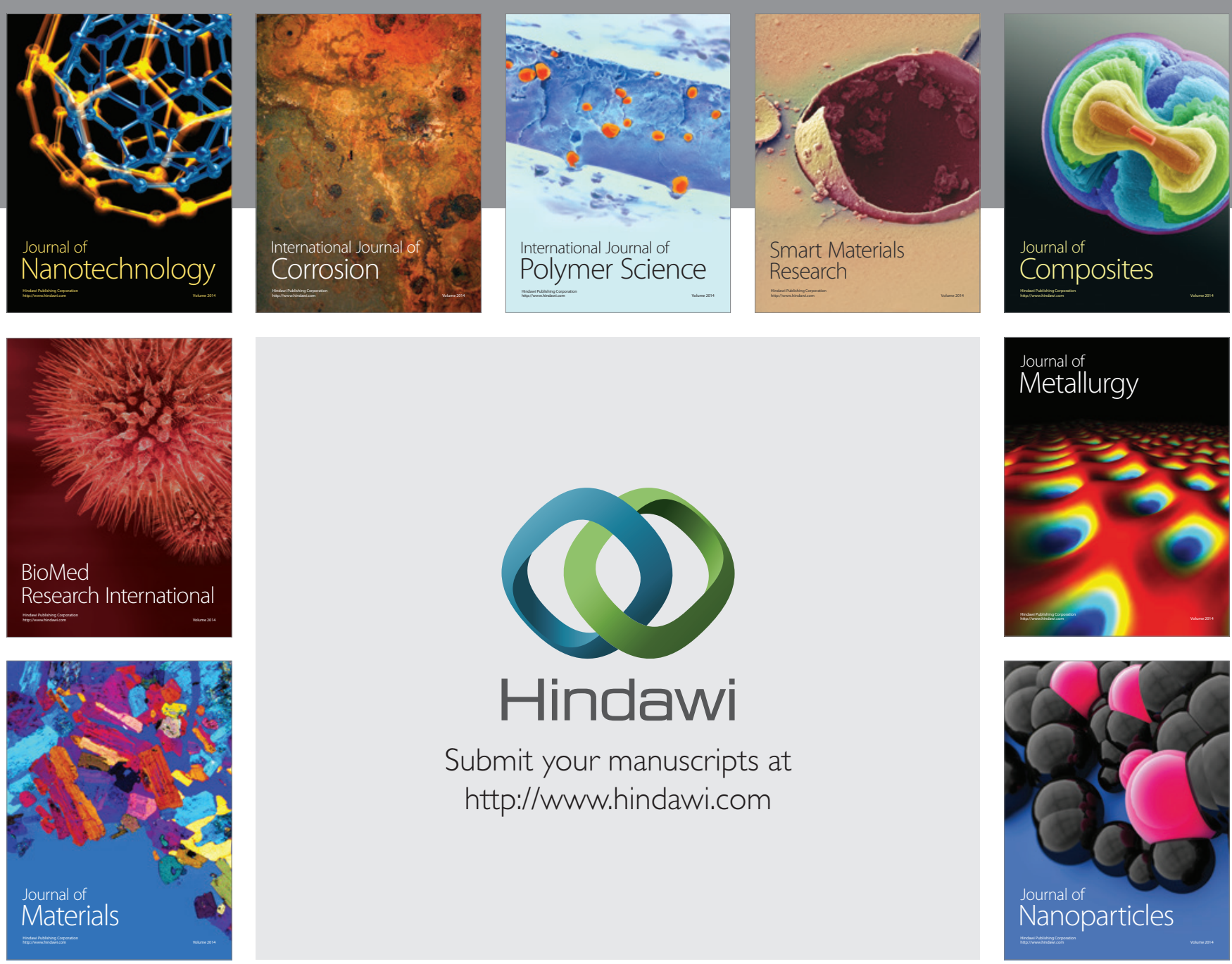

Submit your manuscripts at http://www.hindawi.com
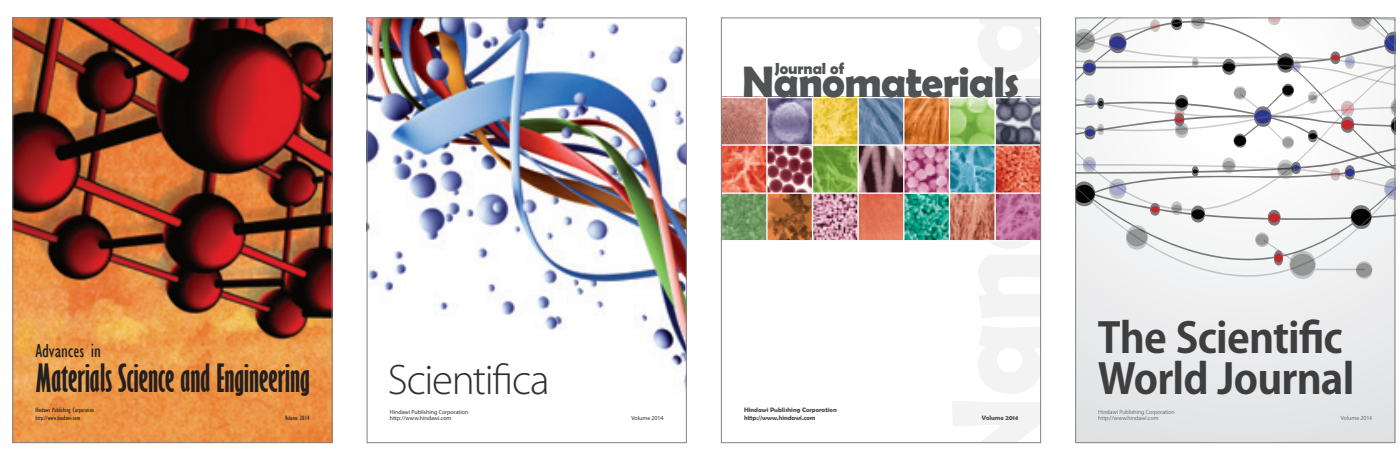

\section{The Scientific World Journal}
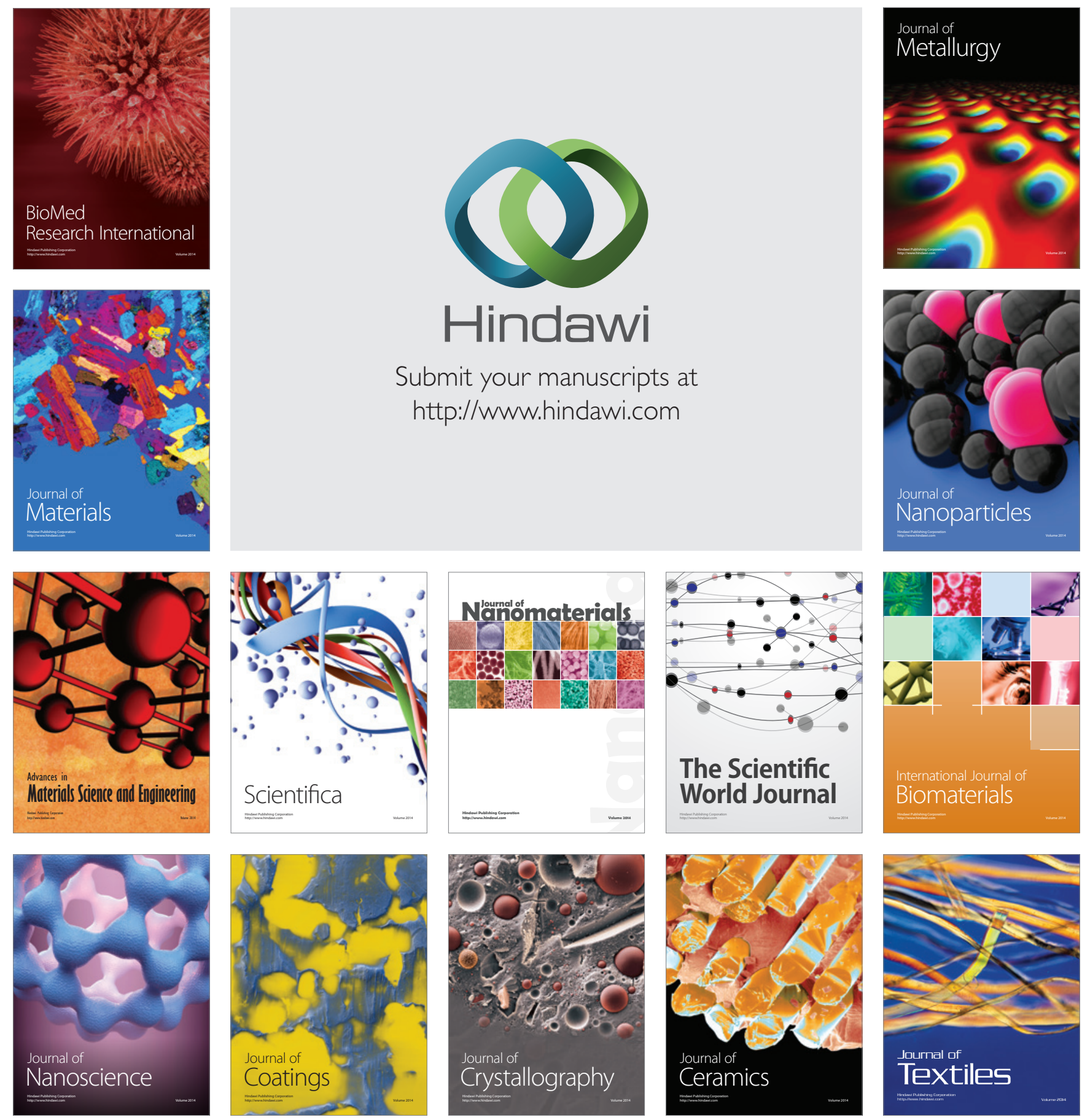